\title{
Transformation of Vestibular Signals for the Control of Standing in Humans
}

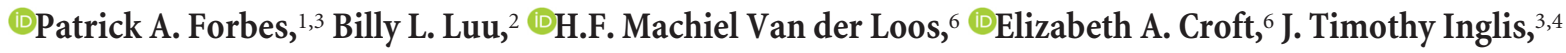 \\ and Jean-Sébastien Blouin ${ }^{3,4,5}$ \\ ${ }^{1}$ Department of Biomechanical Engineering, Faculty of Mechanical, Maritime and Materials Engineering, Delft University of Technology, Delft 2628CD, The \\ Netherlands, ${ }^{2}$ Neuroscience Research Australia, Randwick, New South Wales 2031, Australia, and ${ }^{3}$ School of Kinesiology, ${ }^{4}$ Djavad Mowafaghian Centre for \\ Brain Health, ${ }^{5}$ Institute for Computing, Information and Cognitive Systems, and ${ }^{6}$ Department of Mechanical Engineering, University of British Columbia, \\ Vancouver, British Columbia V6T 1Z1, Canada
}

During standing balance, vestibular signals encode head movement and are transformed into coordinates that are relevant to maintaining upright posture of the whole body. This transformation must account for head-on-body orientation as well as the muscle actions generating the postural response. Here, we investigate whether this transformation is dependent upon a muscle's ability to stabilize the body along the direction of a vestibular disturbance. Subjects were braced on top of a robotic balance system that simulated the mechanics of standing while being exposed to an electrical vestibular stimulus that evoked a craniocentric vestibular error of head roll. The balance system was limited to move in a single plane while the vestibular error direction was manipulated by having subjects rotate their head in yaw. Vestibular-evoked muscle responses were greatest when the vestibular error was aligned with the balance direction and decreased to zero as the two directions became orthogonal. This demonstrates that muscles respond only to the component of the error that is aligned with the balance direction and thus relevant to the balance task, not to the cumulative afferent activity, as expected for vestibulospinal reflex loops. When we reversed the relationship between balancing motor commands and associated vestibular sensory feedback, the direction of vestibular-evoked ankle compensatory responses was also reversed. This implies that the nervous system quickly reassociates new relationships between vestibular sensory signals and motor commands related to maintaining balance. These results indicate that vestibular-evoked muscle activity is a highly flexible balance response organized to compensate for vestibular disturbances.

Key words: electrical vestibular stimulation; postural control; standing balance; vestibular transformations; vestibular-evoked response

Significance Statement

The postural corrections critical to standing balance and navigation rely on transformation of sensory information into reference frames that are relevant for the required motor actions. Here, we demonstrate that the nervous system transforms vestibular sensory signals of head motion according to a muscle's ability to stabilize the body along the direction of a vestibular-evoked disturbance. By manipulating the direction of the imposed vestibular signal relative to a muscle's action, we show that the vestibular contribution to muscle activity is a highly flexible and organized balance response. This study provides insight into the neural integration and central processing associated with transformed vestibulomotor relationships that are essential to standing upright.

\section{Introduction}

The nervous system's ability to encode, decode, and make use of sensory information is essential for navigating and interacting

Received June 3, 2016; revised Sept. 19, 2016; accepted Sept. 20, 2016.

Author contributions: P.A.F., B.L.L., H.F.M.V.d.L., E.C., J.T.I., and J.-S.B. designed research; P.A.F., B.L.L., and J.-S.B. performed research; P.A.F., B.L.L., and J.-S.B. analyzed data; P.A.F. and J.-S.B. wrote the paper.

P.A.F. received funding from the European Union's Seventh Framework Programme for Research, Technological Development and Demonstration under the People Programme (Marie Curie Actions Grant 624158). J.-S.B., J.T.I., E.C., and H.F.M.V.d.L. received operational funding from the Natural Sciences and Engineering Research Council (NSERC) of Canada Discovery Program. B.L.L. received salary support through J.T.I's NSERC Discovery Accelerator within our world. Crucial to this process is the vestibular system, which provides information related to head movement and orientation. A central issue with vestibular signals is that they are
Supplement. We thank Eric Pospisil for contributions to the experimental apparatus, Brandon Rasman for help with data collection, and all subjects who participated in this research.

The authors declare no competing financial interests.

Correspondence should be addressed to Jean-Sébastien Blouin, School of Kinesiology, University of British Columbia, 210-6081 University Boulevard, Vancouver, B.C., Canada, V6T 121. E-mail: jsblouin@mail.ubc.ca. DOI:10.1523/JNEUROSCI.1902-16.2016

Copyright $\odot 2016$ the authors $\quad 0270-6474 / 16 / 3611510-11 \$ 15.00 / 0$ 
encoded in head space and must be transformed into coordinates relevant to ongoing whole-body tasks (such as postural control or navigation). For example, during standing balance, equivalent whole-body movements with different head-on-body positions (e.g., upright vs facing down) will activate different populations of afferents from otolith and semicircular canal end organs but require identical whole-body postural corrections. Likewise, equivalent afferent vestibular signals of head motion may require whole-body movement in different directions depending on where the head is facing. The transformation of vestibular signals in both scenarios must account for the orientation of the head on the body in addition to the contribution of the muscle actions generating the postural response. It is the combination of these two aspects of vestibular transformation during standing that interests us here.

Electrical vestibular stimulation, which distorts the firing rate of vestibular afferents (Goldberg et al., 1984; Kim and Curthoys, 2004), can be used to probe human vestibular function. The isolated vestibular error signal evokes a virtual head rotation about a roll axis fixed in head coordinates (Day and Fitzpatrick, 2005). During standing, this vestibular error evokes a stereotypical pattern of compensatory muscle and whole-body postural responses, with two features suited to investigate the transformation of vestibular signals. First, because the vestibular error is fixed to the head, compensatory responses are transformed according to the alignment of the head relative to the feet (Nashner and Wolfson, 1974; Lund and Broberg, 1983; Mian and Day, 2014). Second, a muscle must be able to contribute to and be relevant for balance control to evoke vestibular compensatory responses. For example, responses are absent when standing subjects, supported by a fixed backboard, either cocontract their ankles or balance a body-equivalent pendulum with their ankles (Fitzpatrick et al., 1994). What would happen if standing balance were limited to a single plane of motion? Because ankle plantar-flexor muscles contribute to balance in anteroposterior and mediolateral planes (Héroux et al., 2014), this condition would satisfy the requirement that muscles contribute and be relevant to postural control to engage the vestibular system in balance. By limiting balance to one plane of motion, however, it is possible to generate a vestibular error signal orthogonal to the plane of motion. Therefore, this would remove the postural relevance of an evoked muscle response to counteract the vestibular disturbance. In our first experiment, we investigate whether vestibular-evoked muscle responses are sensitive to the alignment between the direction of balance (i.e., anteroposterior/mediolateral) and the vestibular error (i.e., head orientation). Using a robotic balance system (Luu et al., 2011), subjects were constrained to one plane of motion while the net direction of the vestibular error was manipulated by having subjects rotate their head in yaw. We hypothesized that a muscle's response to the error would scale with the component of the error that is aligned with the direction of balance.

If, as we hypothesize, the balance system responds selectively to the component of the vestibular error aligned with the balance direction, we then questioned whether it can also adapt the response to a reversal of the vestibular consequences of muscle actions required to stand. For this second experiment, we introduce a novel balancing task by independently controlling body and ankle angles such that vestibular feedback of applied ankle torques was reversed. Because the response to a vestibular error is dependent on the balancing task (Fitzpatrick et al., 1994; Luu et al., 2012), we hypothesized that the balance system would reverse the direction of vestibular-evoked ankle compensatory responses in accordance with the transformation of the sensory expectations of the balance motor actions.

\section{Materials and Methods}

\section{Subjects}

A total of 22 healthy subjects (all males; age $28.3 \pm 5.0$ years, mean \pm SD) with no self-reported history of neurological disorders participated in this study. The experimental protocol was explained before the experiment and all subjects gave written informed consent. The experiment conformed to the Declaration of Helsinki and was approved by the University of British Columbia's Clinical Research Ethics Board.

\section{Experimental setup}

Three experiments were conducted to study the transformation of vestibular signals of head motion for the control of standing balance in humans. In Experiments 1A and 1B, the vestibulo-motor transformation was examined by limiting the balancing task to a single plane of motion (anteroposterior or mediolateral) with head yaw postures ranging from a head over-the-left-shoulder to a head-forward position. In Experiment 2 , we then examined the vestibulo-motor transformation by reversing the vestibular sensory consequences of normal balancing motor actions.

For all experiments, subjects stood on a robotic balance system programmed with the mechanics of an inverted pendulum to simulate the load of the body during standing (Fig. 1A). The robotic balance system consists of a whole-body motion platform (6DOF2000E; MOOG) to control the orientation of the subject's body and a custom ankle-tilt platform to independently control the pitch of the ankles (Fig. 1A). Subjects stand on two force plates (BP250500; AMTI) attached to the ankletilt platform with their body strapped to a backboard mounted on the whole-body motion platform. Seatbelts placed across the shoulders and around the waist prevent subjects from falling forward without supporting the load of the body acting through the feet. A real-time system (PXI-8196; National Instruments) operating at $60 \mathrm{~Hz}$ controls the whole-body motion platform (6DOF2000E; MOOG) while a second real-time system (PXI-7350; National Instruments) operating at $200 \mathrm{~Hz}$ controls the ankle-tilt platform. The entire system has a delay of $\sim 41.5$ ms between the position command and the measured position of both the whole-body and ankle-tilt platforms. A detailed description of the setup and design of the robotic balance system can be found in Luu et al. (2011) and Pospisil et al. (2012). Briefly, the inverted pendulum was modeled to match the physical dimensions of each subject's body (i.e., measured mass and center of mass height) using a distributed-mass model. The distributed-mass model was chosen over a concentratedmass model because it better represents the load stiffness of the human body during normal standing (Luu et al., 2011). Ankle torques measured via the force plates are used as input to the inverted pendulum model to stabilize the robotic balance system in both the anteroposterior and mediolateral planes.

In the anteroposterior plane, the whole-body motion platform and ankle-tilt platform rotated about an axis that passed through the ankle joints. The combination of the whole-body and ankle-tilt platforms ensured that the body and foot angles could be manipulated independently by modifying the gains of the ankle-torque/body-angle or ankle-torque/ ankle-angle relationships. For this study, the gains were chosen to simulate balance in two conditions. The first condition was used in Experiments 1A, 1B, and 2 (see "Experimental protocol" section) and replicated normal human standing wherein the whole-body motion platform rotated the subject's body and the ankle-tilt platform counterrotated to simulate an earth-fixed support surface. In a forward leaning position, plantar-flexor torque measured by the force plates was required to stabilize the robotic balance system in the same way ankle torque must stabilize the body during normal over-ground standing (Fig. 1B). In this case, an increase in plantar-flexor torque greater than the torque due to gravity will cause the body to rotate backward. The second condition was used in Experiment 2 (see "Experimental protocol" section) and was designed to reverse the vestibular consequences of ankle actuation. To simulate this on the robotic balance system, the ankle-torque/bodyangle relationship was reversed (i.e., a gain of -1 ) while the normal ankle-torque/ankle-angle relationship was maintained (i.e., a gain of 1). In a rearward leaning position, plantar-flexor torque measured by the force plates was required to stabilize the robotic balance system, thus reversing the vestibular consequences (i.e., feedback) of the ankle torques 


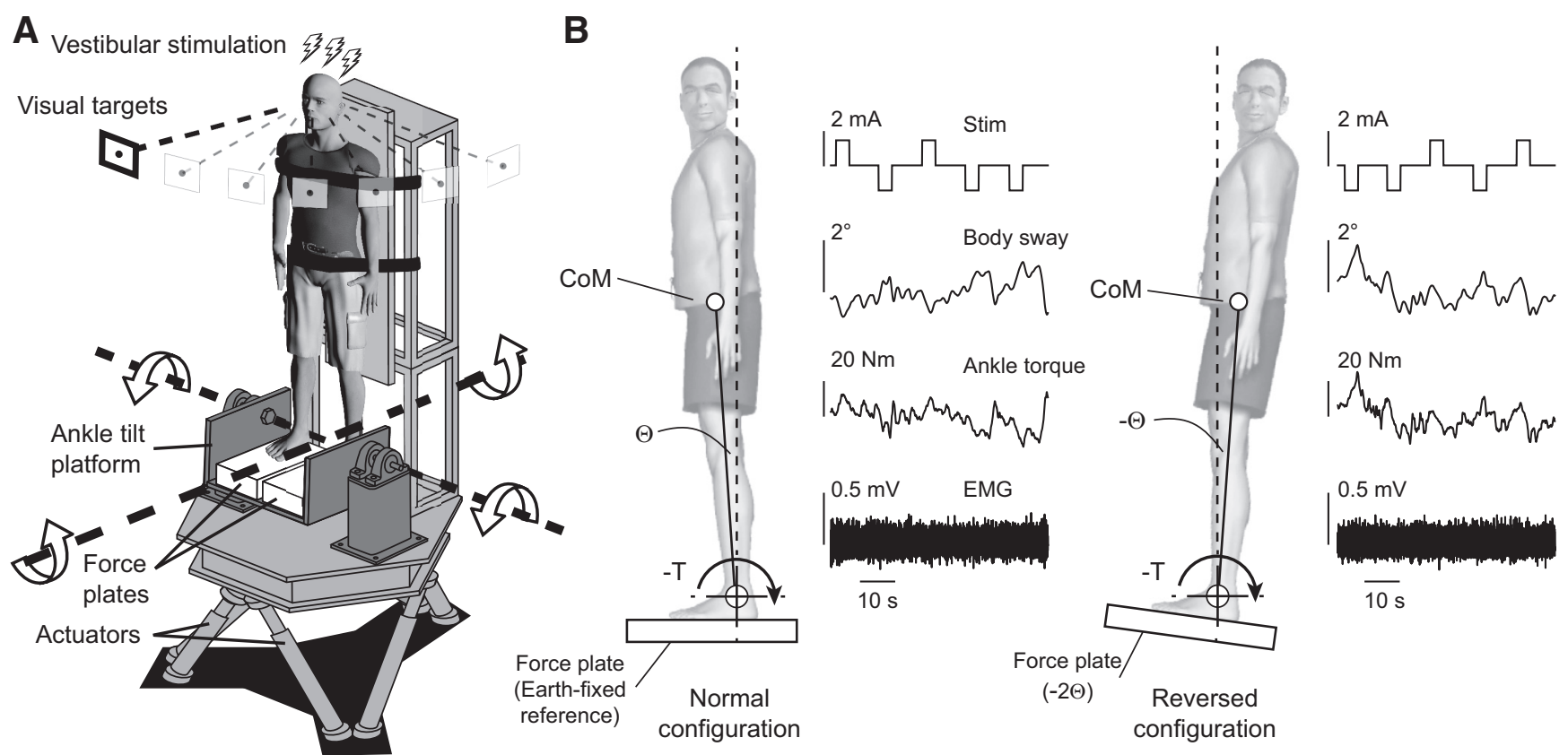

Figure 1. Experimental setup. $\boldsymbol{A}$, The subject stood on an ankle-tilt platform (dark gray components) and was securely strapped to a rigid backboard mounted on top of a whole-body motion platform (light gray components). Both the ankle-tilt platform and whole-body motion platform were controlled by modulating the sum of the ankle torques exerted on the force plates. The subject rotated either in the anteroposterior plane or the mediolateral plane. In the anteroposterior plane, both platforms rotated about an axis that passed through the subject's ankles (dashed line) with the ankle-tilt platform counter-rotating relative to the motion platform to maintain a level foot orientation. In the mediolateral plane, only the whole-body motion platform rotated about an axis midway between the subject's ankles (dashed line). $\boldsymbol{B}$, The subject balanced the robotic balance system with either normal or reversed vestibular feedback in the anteroposterior plane while keeping their eyes closed. In the normal condition, plantar-flexor torque was necessary to maintain the body in a forward leaning position. In the reversed condition, plantar-flexor torque was necessary to maintain the body in a rearward leaning position, thus reversing the vestibular consequences of the motor output. $\Theta$ is the angle of the body's center of mass in space, CoM is the body's center of mass, and $\mathrm{T}$ is the torque generated around the ankle joint required to keep the body upright. We choose as a convention to report the torque applied to the body. To maintain a normal ankle-torque/ankle-angle (i.e., somatosensory) relationship, the ankle-tilt platform rotated in the same direction as body sway at twice the magnitude. In this way, we limit our modifications only to a reversal of the vestibular consequences of the ankle torques required to stand. Raw data of the vestibular stimulus, body sway, ankle torque, and EMG activity of the right soleus muscle are shown during a galvanic vestibular stimulation trial (see "Stimuli" section) in which the subject balanced the robotic balance system programmed with the mechanics of an inverted pendulum with normal and reversed vestibular feedback.

required to stand (Fig. $1 B$ ). In this case, an increase in plantar-flexor torque greater than the torque due to gravity will cause the body to rotate forward. To ensure that the ankle somatosensory feedback signals produced by the balancing ankle torques were not altered (i.e., maintaining the same ankle-torque/ankle-angle relationship across trials with normal and reversed vestibular feedback), the ankle tilt platform was rotated in the same direction as body sway at twice the magnitude. As a result, the foot was not held horizontal in trials in which the vestibular feedback was reversed. For example, when subjects maintained a backward leaning posture, the ankle-tilt platform was in a toe-up orientation with an angle twice the backward body angle (Fig. 1B).

In the mediolateral plane, the whole-body motion platform rotated about a midpoint between the ankles. As the whole-body motion platform rotates rightward, a corrective net torque comprised of both vertical forces and ankle torques obtained from both plates must be applied to stabilize the simulated body. This balance condition was used for Experiment 1B (see "Experimental protocol" section). Because the ankle joints are sway referenced to the motion of the whole-body motion platform, the normal contributions of passive stiffness and damping arising from various sources (i.e., the ankles and hips) during mediolateral ankle motion were simulated for each subject according to Luu et al. (2011) using ankle properties derived from the anteroposterior direction (Loram and Lakie, 2002).

Angular motion of the body was limited to a maximum sway of $6^{\circ}$ anterior and $3^{\circ}$ posterior during trials in which subjects balanced in the anteroposterior plane (Experiments 1A and 2) with normal vestibular feedback. These sway limits were inverted for trials with reversed vestibular feedback (Experiment 2). For trials involving mediolateral balance (Experiment 1B), maximum body motion was limited to $\pm 4^{\circ}$. These limits ensured that the subject could generate sufficient torque to balance the robotic balance system across the range of motion.
Stimuli. We used two forms of electrical vestibular stimuli to modulate the firing rate of the vestibular nerve (Goldberg et al., 1984; Kim and Curthoys, 2004): stochastic vestibular stimulation (SVS) signals and squarewave galvanic vestibular stimulation (GVS) pulses. Both stimuli provide an isolated craniocentric vestibular error signal, which evokes an illusory or "virtual" sensation of movement without feedback from other sensory modalities. The behavioral responses evoked by the error signal have been modeled based on afferent populations within the labyrinth and the assumption that both otoliths and semicircular afferents are affected by the electrical current (Fitzpatrick and Day, 2004). When delivered in a binaural bipolar arrangement, the electrical stimulus evokes primarily a sensation of head roll rotational velocity (Peters et al., 2015) about an axis directed posteriorly and superiorly by $18^{\circ}$ relative to the Reid plane (Fitzpatrick and Day, 2004; St George et al., 2011). Although electrical vestibular stimulation activates both canal and otolith afferents (Kim and Curthoys, 2004), the primary postural response is due to this head roll while linear responses driven by otolithic signals are relatively small (Day and Cole, 2002; Wardman et al., 2003; Mian et al., 2010). The near symmetry of afferent populations across the macular striola of the utricles (Tribukait and Rosenhall, 2001) is predicted to result in a near cancellation of a net otolithic output during electrical stimulation applied to the mastoid processes (Fitzpatrick and Day, 2004). When standing, this virtual vestibular error signal has a strong effect on balance control and evokes compensatory muscle and whole-body responses that help to maintain upright stance (Day et al., 1997). Here, we were interested in using the consistent nature of the evoked muscle and whole-body responses to examine the transformation of vestibular signals of head motion for the control of standing balance in humans.

SVS signals were designed with a bandwidth of $0-25 \mathrm{~Hz}$ and peak amplitudes of either $\pm 4 \mathrm{~mA}$ [Experiments $1 \mathrm{~A}$ and $1 \mathrm{~B}$; root mean square (RMS) $0.85 \mathrm{~mA}$ ] or $\pm 5 \mathrm{~mA}$ (Experiment 2; RMS $1.51 \mathrm{~mA}$ ). The larger RMS of the SVS signal used in Experiment 2 was intended to increase the 


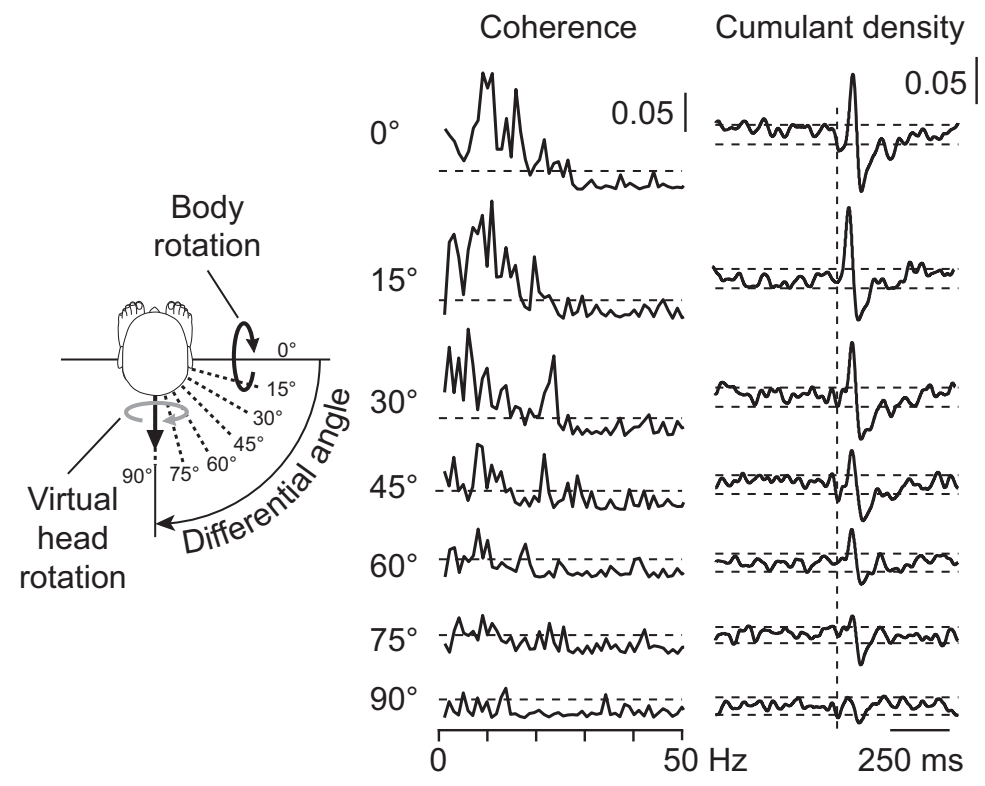

Figure 2. Vestibular-evoked muscle responses for a single subject at all differential head angles. Coherence and cumulant density are calculated between the vestibular stimulus and soleus EMG activity. Horizontal lines represent the $95 \%$ confidence limits. The vertical line in the cumulant density plot indicates the zero lag mark between the SVS and muscle activity.

signal power and evoke a larger muscle response (Forbes et al., 2014). Square-wave GVS pulses (Experiment 2) were designed with a duration of $4 \mathrm{~s}$ and an amplitude of $1.5 \mathrm{~mA}$; the anode right/cathode left (ARCL) and anode left/cathode right (ALCR) polarity arrangements were presented randomly with an interstimulus interval varying between 7 and $11 \mathrm{~s}$. This interstimulus duration ensured that subjects had sufficient time to return to their normal upright balanced position after stimulus delivery and subsequent sway.

The electrical stimuli were delivered to subjects using carbon rubber electrodes $\left(\sim 9 \mathrm{~cm}^{2}\right)$ in a binaural bipolar arrangement. The electrodes were coated with Spectra 360 gel (Parker Laboratories) and secured behind the mastoid processes using tape. The stimuli were delivered as analog signals via a data acquisition board (PXI-6289; National Instruments) to an isolated constant current stimulator (STMISOL; Biopac). The signals were generated offline using LabVIEW (Experiments 1A and 1B; National Instruments) or MATLAB software (Experiment 2; The MathWorks).

Data recordings. Electromyography (EMG) and electrical vestibular stimulation signals were recorded on a real-time data acquisition board (PXI-6289; National Instruments) at $2000 \mathrm{~Hz}$. EMG activity of the right soleus muscle was measured using $\mathrm{Ag}-\mathrm{AgCl}$ surface electrodes (Blue Sensor M; Ambu) after being amplified $(1000 \times)$ and filtered between 30 and $1000 \mathrm{~Hz}$ (P55 AC amplifier; Grass Technologies). Whole-body motion platform angle (i.e., body sway), obtained from position encoders and force plate data were recorded at $2000 \mathrm{~Hz}$ using a second data acquisition board (PXI-6229; National Instruments) and synchronized with EMG and SVS data in real time.

\section{Experimental protocol}

Subjects were given visual targets that were placed in an arc $\sim 2 \mathrm{~m}$ away from them. A laser pointer attached to the subject's head was used to align and maintain the head's orientation at a desired head yaw position and tilted in extension such that the Reid plane was angled $18^{\circ}$ up from horizontal. This head position aligns the estimated axis of virtual vestibular error signal evoked by the vestibular stimulus with earth horizontal and maximizes the postural responses to binaural bipolar electrical vestibular stimulation in a direction aligned with the interaural axis (Fitzpatrick and Day, 2004; Cathers et al., 2005; Day and Fitzpatrick, 2005). Electrical vestibular stimulation was applied in all trials to probe the vestibular control of standing, as explained in the sections below. The experiments were performed on three separate days, each completed in one session. A total of 22 subjects were recruited: nine participated in
Experiment 1A; 12 participated in Experiment 1B (of whom seven had participated in Experiment $1 \mathrm{~A}$ and one had participated in Experiment 2); and eight participated in Experiment 2.

Experiment 1A. Experiment $1 \mathrm{~A}$ assessed whether the balance system responds selectively to the component of the vestibular error that is aligned with the direction of balance. If it does, then we would expect a minor or absent muscle response when the direction of the vestibular error does not align with the direction of balance. Subjects stood with their eyes open and supported upright on top of the robotic balance system, which was programmed to move only in the anteroposterior plane. They directed their heads toward seven targets located at $15^{\circ}$ increments between an over-theleft-shoulder position and a head-forward position. Each trial was performed at a different head angle that was held actively for the duration of the trial. The angle between the direction of balance stabilization and the direction of the electrically evoked vestibular error signal of head rotation is defined here as the differential head angle; that is, the angular difference between the body rotation vector and the vestibular error vector (Fig. 2). Starting with the head over the left shoulder position, the differential head angle changed from $0^{\circ}$ to $90^{\circ}$ when the head was in the forward position. For each differential head angle, subjects were exposed to stochastic vestibular stimulation for 3 min while they were balancing the robotic balance system. The amplitude of the vestibular-evoked muscle response was used for comparison across the seven head orientations. Trial order for the differential head angle was randomized for each subject and subjects were free to move their heads between trials.

Experiment $1 B$. It is possible that the influence of head yaw orientation on the response amplitude is because the soleus muscle does not respond to balance disturbances (i.e., a vestibular error) directed in the mediolateral plane. Given that the soleus contributes to normal balance in both planes of motion (Héroux et al., 2014), this possibility was thought to be unlikely. However, we wanted to confirm this assumption because the additional constraint imposed by limiting sway to a single plane may have modified the normal contribution of the muscle during these conditions. Nine subjects stood with their eyes open and supported upright on top of the robotic balance system with the head forward while balancing in either the mediolateral or anteroposterior plane only. For both trials, subjects were exposed to stochastic vestibular stimulation for $3 \mathrm{~min}$ and trial order was randomized for each subject. The presence of a response when balancing in the mediolateral plane would indicate that the soleus muscle can respond to a vestibular error that perturbs balance in a mediolateral plane. When balancing in the anteroposterior plane, however, we expected that the soleus muscle would not respond to the vestibular error because it is directed orthogonally to the direction of balance.

With the ankle joints sway referenced to whole-body motion during mediolateral trials, it may be possible that there is an increased reliance on vestibular information in the absence of somatosensory information (Cenciarini and Peterka, 2006) relative to the normal ankle motion provided in anteroposterior trials. This could result in a smaller evoked response during anteroposterior balance due to a reduced reliance on vestibular information because reliable somatosensory cues are available. Therefore, we performed an additional control experiment in which five subjects (of whom one was from the first group of nine subjects) balanced in the anteroposterior plane with and without the ankles being sway referenced. Here, we expected that sway referencing would have a minimal effect on the vestibular-evoked response in the soleus muscle because the direction of the vestibular error is orthogonal to the balance direction for both conditions.

Experiment 2. Experiment 2 assessed whether reversing the vestibular consequences (i.e., feedback) of the ankle torques required to stand also 
reverses the compensatory motor responses evoked by a vestibular error signal. Subjects were supported upright in the robotic balance system with their head turned to the left for all trials. They were required to keep the body balanced and upright in space when the vestibular consequences of ankle-balancing torque were either normal or reversed. Subjects were instructed to lean slightly forward during conditions with normal vestibular feedback and lean slightly backward during conditions with reversed vestibular feedback. In this way, both conditions engaged the soleus muscle in the balancing tasks. A possible limitation to this approach is that, across the normal and reversed configurations, the opposing leaning postures (i.e., $\sim 1-2^{\circ}$ forward and rearward, respectively) generate differing head roll positions and static otolith activity, which may influence the expected reversal of the vestibular-evoked response. This is unlikely, however, because the polarity of postural responses evoked by electrical vestibular stimulation during standing balance remains unchanged for variations in head roll up to $\pm 90^{\circ}$ (Marsden et al., 2002; Reynolds, 2011).

When subjects were first exposed to the condition with reversed vestibular feedback, they were given a minimum of $30 \mathrm{~s}$ to familiarize themselves with this novel balance task and assume a stable upright posture. Because ankle feedback was not altered, subjects were also instructed to close their eyes, thus isolating the reversal of sensorimotor relationships to vestibular feedback only.

A total of eight trials were performed, four per condition (i.e., normal vs reversed vestibular feedback), and the condition order was randomized across subjects. Within both the normal and reversed conditions, 3 of the trials exposed subjects to square-wave GVS pulses and one trial exposed subjects to SVS for $3 \mathrm{~min}$. Square-wave GVS trials were performed in addition to SVS trials to evoke a well defined postural response (i.e., torque and body sway) to the electrical stimulation by applying sustained currents (i.e., $4 \mathrm{~s}$ at $\pm 1.5 \mathrm{~mA}$ ). Each square-wave trial (3 per condition) consisted of 16 pulses ( 8 anode right/cathode left and 8 cathode right/anode left) for a total of 24 repetitions per stimulus polarity and vestibular feedback (normal vs reversed) combination. SVS trials were performed to evaluate the soleus muscle response to electrical vestibular stimulation across the two vestibular feedback conditions in the frequency (phase and coherence) and time (cross-covariance) domains (see "Measurement and signal analysis" section). We opted to use SVS instead of GVS to evaluate the muscle response to limit the experimentation time; GVS requires a substantial number of repetitions to achieve equivalent signal-to-noise ratios compared with SVS (Dakin et al., 2007; Reynolds, 2011), which would make our experiment prohibitively long. Furthermore, SVS allowed us to provide a phasefrequency estimate between the stimulation signal and muscle activity, which provides additional information regarding the timing and polarity of the evoked muscle activity not available using square-wave GVS pulses. When the vestibular feedback was reversed, the SVS trial was performed immediately after the $30 \mathrm{~s}$ familiarization period. Here, we examined the SVS- and GVS-evoked balance responses across conditions with normal and reversed vestibular feedback of balancing motor actions.

\section{Signal analysis}

Coherence, phase, and cumulant density functions were calculated within each participant with the data from each SVS trial to evaluate the correlation between the input electrical stimulus and the rectified EMG (Dakin et al., 2014). Subject data were then averaged across all subjects to provide grouped means. Coherence- and phase-frequency estimates were calculated using a window of 2048 points $(\sim 1 \mathrm{~s})$ to give a resolution of $\sim 0.98 \mathrm{~Hz}$. Coherence is used as a measure of the linear relationship between the two signals at the frequencies considered (Pintelon and Schoukens, 2012) and is significant when exceeding the $95 \%$ confidence limit as derived from the number of disjoint segments (Halliday et al., 1995). Phase was used as a measure of timing between the input stimulus and muscle activity and was estimated at frequencies exhibiting significant coherence. Cumulant density functions were derived by taking the inverse Fourier transform of the cross-spectrum (Halliday et al., 1995) and then normalized (between -1 and +1 ) by the product of the vector norms of the input and output signals (Dakin et al., 2010). Cumulant density responses represent the time-domain correlation (i.e., crosscovariance) between SVS and muscle activity and were used throughout this study to assess the magnitude of the vestibular-evoked muscle response. In the lower limb muscles, the cumulant density exhibits a biphasic pattern with two opposing peaks defined as the short $(50-70 \mathrm{~ms})$ and medium (100-120 ms) latency peaks (Nashner and Wolfson, 1974; Britton et al., 1993; Fitzpatrick et al., 1994; Fitzpatrick and Day, 2004; Dakin et al., 2007; Dakin et al., 2011). For comparison across conditions, the timing and direction of both peaks, as well as the peak-to-peak amplitude, were extracted from each subject's response. When either of the two peaks did not exceed the $95 \%$ confidence limit, this subject's response was considered absent and its peak-to-peak amplitude was set to zero. This was done to avoid ambiguous identification of the short- and medium-latency peaks, which are similar in magnitude to the surrounding noise when they fall below the confidence limit.

Body sway and anteroposterior torque during square-wave trials were trigger averaged to the GVS pulse onset. Trigger-averaged sway responses are characterized by sway of the body toward the anode (Lund and Broberg, 1983; Britton et al., 1993; Day et al., 1997). From the individual subject averages, the peak sway response was extracted for each vestibular feedback condition and stimulus polarity. Trigger-averaged ankle torque profiles, in contrast, are composed of two specific features: (1) an initial short duration (from 0 to $1 \mathrm{~s}$ ) peak that accelerates the body in the direction of the anode and (2) a sustained and larger response that prevents the body from toppling over (Day et al., 1997). We extracted the initial torque peak over a window of $0-1 \mathrm{~s}$ after stimulus onset to assess any reversal in the vestibular-evoked motor response that initiates body sway. Finally, as a general assessment of balancing behavior, we extracted the mean body angle and mean-removed RMS of body sway during the two vestibular feedback conditions. Both mean body angle and RMS body sway were calculated over the entire length of the three GVS trials and from the SVS trial. All data were averaged across subjects to provide group means and a $95 \%$ confidence interval.

\section{Data reduction}

To test our first hypothesis that the vestibular-evoked response would scale with the component of the vestibular error signal aligned with the direction of balance stabilization (Experiment 1A), we first evaluated the effect of head position on the amplitude of vestibular-evoked muscle responses using a repeated-measures ANOVA. We then performed a nonlinear regression of the subject average vestibular-evoked responses to determine whether the variation could be explained by a cosine function using the differential head angle (i.e., difference between the vestibular error and balance directions). The goodness-of-fit for the estimated cosine function was evaluated using the coefficient of determination $\left(R^{2}\right)$. We also compared the vestibular-evoked responses across the two directions of balance (anteroposterior and mediolateral) with the head forward (Experiment 1B) using a Student's $t$ test. We expected that, when balancing in the anteroposterior plane with the head forward, the vestibular-evoked responses (and coherence) would not exceed the $95 \%$ confidence limit because the vestibular error was orthogonal to the balance direction. In contrast, we expected that, when balancing in the mediolateral plane, the vestibular-evoked responses would exceed the $95 \%$ confidence limit. To control for potential effects of sway referencing, we examined the muscle responses during anteroposterior balance with and without sway referencing while subjects maintained their head forward. We expected that the vestibular-evoked responses (and coherence) would not exceed the $95 \%$ confidence limit in either condition (i.e., with and without sway-referencing) because the vestibular error was orthogonal to the balance direction.

To test our second hypothesis that vestibular-evoked balance responses are inverted when reversing the vestibular consequences of ankle-balancing torque, we first compared the GVS-evoked peak body sway and initial peak torque across the two vestibular feedback conditions. Student's $t$ tests were performed to compare peak body sway across conditions within each stimulus polarity. Wilcoxon-paired signed-rank tests were performed to compare initial peak torque within each stimulus because these responses were not distributed normally. We expected that, because the electrically evoked vestibular error signal is identical for both the normal and reversed vestibular feedback conditions, the evoked sway would be in the same direction for a given stimulus polarity. How- 


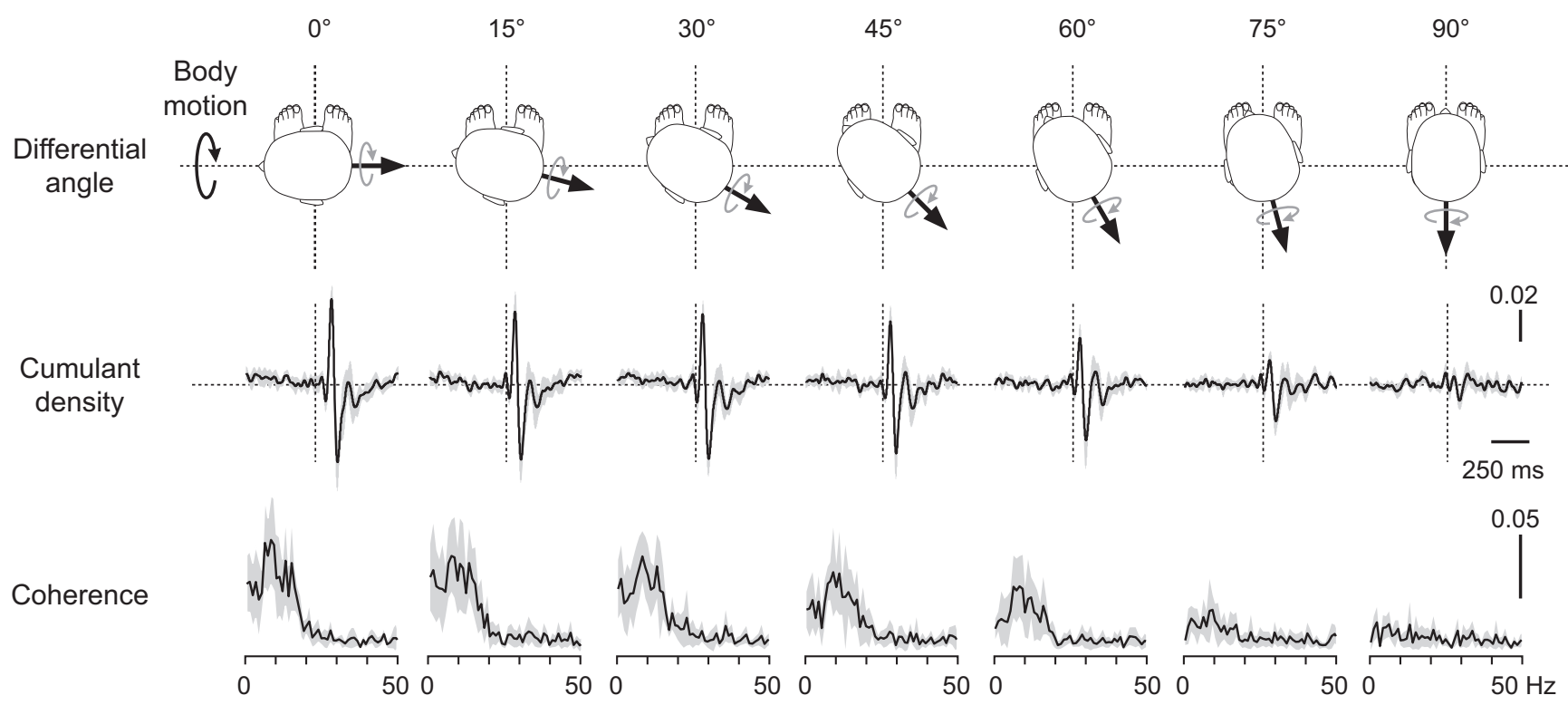

Figure 3. Group mean $(n=9)$ muscle responses to electrical vestibular stimulation at all differential head angles. For illustrative purposes, shaded areas represent the $95 \%$ confidence intervals about the group mean for the coherence and cumulant density functions. Vertical lines in the cumulant density plots indicate the zero lag mark between the SVS and muscle activity.

ever, to generate this equivalent direction of sway across our conditions, we expected that the evoked torque response would be reversed. To determine whether vestibular-evoked muscle responses were also inverted when reversing the vestibular feedback, we examined the direction of the two SVS-evoked muscle response peaks (short and medium latency) visually in all subjects. The muscle response evoked by SVS can generate spurious oscillations in the cumulant density estimates before the typical short- and medium-latency peaks (Dakin et al., 2007; Mian et al., 2010; Reynolds, 2010; Dakin et al., 2011; Luu et al., 2012; Forbes et al., 2014) and can lead to ambiguity when interpreting the timing and direction of our results. Therefore, to provide further confirmation that the evoked muscle response was inverted, we also compared the mean SVSEMG phase-frequency estimate across our two conditions: an inverted response would be observed as a $180^{\circ}$ shift in the phase-frequency muscle estimate. Finally, because most subjects had never been exposed to balance conditions with reversed vestibular feedback, we evaluated any changes in general balancing behavior across the normal and reversed vestibular feedback conditions. For this, we compared the absolute value of the mean body angle and mean-removed RMS body sway during both GVS and SVS trials using Student's $t$ tests. Furthermore, because changes in sway can modify the magnitude of the electrically evoked muscle response (Fitzpatrick et al., 1994; Bacsi and Colebatch, 2005), we also compared SVS-evoked peak-to-peak amplitude across our two vestibular feedback conditions using a Student's $t$ test. All statistical analyses were performed using SPSS22 (IBM) and significance was set at the 5\% level.

\section{Results}

Effect of head orientation and balance direction on the vestibular-evoked muscle responses (Experiments 1A and 1B) All subjects were able to stabilize the balance robot in both the anteroposterior and mediolateral planes in all head positions without difficulty. Data from a representative subject when balancing in the anteroposterior plane at different head yaw orientations (Experiment 1A) are shown in Figure 2. With the head facing left (i.e., differential head angle of $0^{\circ}$ ), the coherence was significant at frequencies up to about $20 \mathrm{~Hz}$. The associated biphasic muscle response evoked by the vestibular stimulation was largest at this head position, with typical short-latency ( $66.5 \mathrm{~ms})$ and medium-latency (103.5 ms) peaks exceeding the $95 \%$ confidence interval. As the head rotated forward, increasing the difference between body stabilization and the vestibular error directions, both the coherence and the evoked

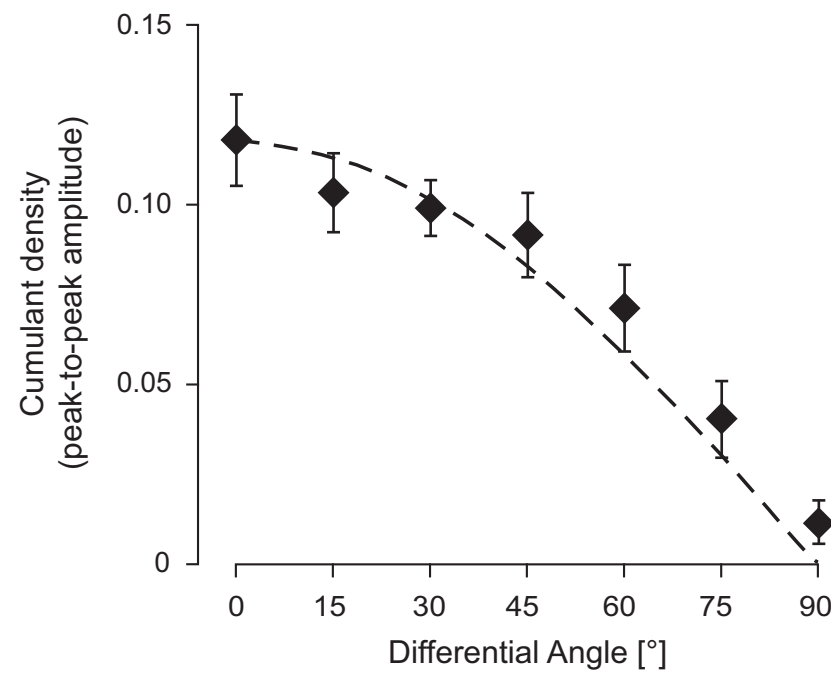

Figure 4. Averaged cumulant density peak-to-peak amplitudes plotted relative to differential head angle. Group mean $(n=9)$ data are plotted together with the cosine transformation of the $0^{\circ}$ responses (dashed line). The measured responses bear a remarkable likeness to the cosine transformed data $\left(R^{2}=0.94\right)$. Error bars indicate the $95 \%$ confidence interval.

muscle response diminished progressively. A similar trend was also observed in the group data (Fig. 3): both coherence and the evoked muscle response were largest at $0^{\circ}$ and decreased with increasing differential head angle. At $75^{\circ}$ and $90^{\circ}$, only six and three subjects, respectively, showed a vestibular-evoked response that exceeded the $95 \%$ confidence limit. Analysis of the data from all subjects revealed a significant effect of head orientation in the vestibular-evoked muscle responses $\left(F_{(6,48)}=44.36, p<0.001\right)$.

The relationship between the peak-to-peak evoked muscle response and the differential head angle was well represented by a cosine function estimate and yielded a high coefficient of determination $\left(R^{2}=0.94\right.$; Fig. 4$)$. These data suggest that, as the head rotates forward, the amplitude of the vestibular-evoked muscle response scales with the component of the vestibular error vector parallel to the direction of body motion. This result differs from the well estab- 


\section{Coherence [-]}



Cumulant density [-]

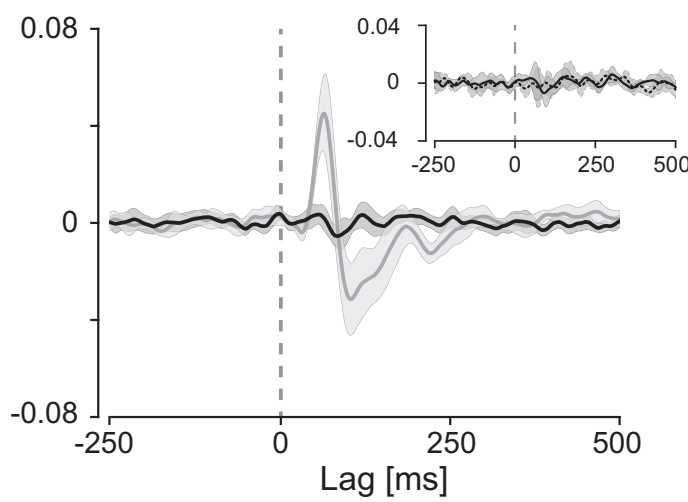

Figure 5. Group mean $(n=9)$ muscle responses to electrical vestibular stimulation during anteroposterior and mediolateral balance trials, both with the head forward. Group mean ( $n=5$ ) muscle responses for the control experiment, which compared head forward anteroposterior balance trials with and without sway referencing, are included as insets. For illustrative purposes, shaded areas represent the $95 \%$ confidence intervals about the group mean for the coherence and cumulant density functions. The vertical line in the cumulant density plot indicates the zero lag mark between the SVS and muscle activity.

lished observations that vestibular-evoked motor responses rotate with the orientation of the head relative to the feet (Nashner and Wolfson, 1974; Lund and Broberg, 1983; Iles and Pisini, 1992; Britton et al., 1993; Pastor et al., 1993; Dakin et al., 2007). Instead, our results are comparable to the reduction of postural responses to vestibular stimulation in the mediolateral plane without changes in the anteroposterior plane when stance width is increased to make the body more stable in the mediolateral plane (Mian and Day, 2014).

When subjects balanced in the mediolateral plane with the head forward (Experiment 1B), coherence was significant at frequencies up to $20 \mathrm{~Hz}$ and the vestibular-evoked muscle responses demonstrated the expected biphasic waveform (Fig. 5). In contrast, when subjects balanced in the anteroposterior plane with the head forward, coherence was near zero and vestibular-evoked muscles responses were almost absent; for six of the nine subjects in this head-forward condition, the vestibular-evoked muscle response did not exceed the $95 \%$ confidence interval. The mean peak-to-peak amplitude of the vestibular-evoked muscle response when balancing in the mediolateral plane was $751 \%$ larger relative to balancing in the anteroposterior plane $\left(t_{(8)}=6.461, p<0.001\right)$. During the anklesway-referenced condition, no subject exhibited evoked muscles responses (or coherence) exceeding the 95\% confidence interval during anteroposterior balance (Fig. 5, inset), eliminating ankle somatosensory cues as a contributing factor for the scaling of muscle responses observed between the anteroposterior and mediolateral trials. These data confirm that, although the soleus muscle is able to respond to a vestibular error that perturbs balance in the mediolateral direction, a response is not evoked when the vestibular error is orthogonal to the direction of balance (i.e., the differential head angle is $90^{\circ}$ ). Furthermore, the results of both experiments indicate that the vestibular-evoked responses correct only for the net signal of head motion that is relevant to (i.e., aligned with) the ongoing balance task.

\section{Effect of reversing the vestibular consequences of balance on the vestibular-evoked postural responses (Experiment 2)}

When we reversed the vestibular consequences of the ankle motor commands required to stand, most subjects were able to stabilize the robotic balance system within the $30 \mathrm{~s}$ familiarization period; however, one subject required an additional minute before being able to sustain a stable upright posture. All subjects took on a backwardleaning posture by generating a plantar-flexor torque (Fig. $1 B$ ), thus engaging the soleus muscle in the balance task. The absolute value of mean body angle was similar across reversed and normal vestibular feedback conditions during both GVS and SVS trials (GVS trials: $1.57 \pm 0.70^{\circ}$ vs $1.46 \pm 0.60^{\circ}$; SVS trials: $1.81 \pm 1.15^{\circ}$ vs $1.53 \pm 1.03^{\circ}$; both $\left.t_{(7)}<0.62, p>0.557\right)$. There was, however, a $63 \%$ and $146 \%$ increase in the mean-removed RMS of sway with reversed vestibular feedback relative to the normal vestibular feedback during GVS and SVS trials, respectively (GVS trials: $0.75 \pm 0.35^{\circ}$ vs $1.23 \pm 0.54^{\circ}$; SVS trials: $0.62 \pm 0.35^{\circ}$ vs $1.53 \pm 0.86^{\circ}$; both $t_{(7)}>3.04, p<0.019$ ).

Square-wave GVS pulses evoked robust body sway and ankle torque responses during trials with normal and reversed vestibular consequences of ankle-balancing torque. Data from a representative subject are shown in Figure 6A. GVS-evoked sway was in a similar direction and magnitude across the two balance conditions for each stimulus polarity. In contrast, GVS-evoked ankle torque responses were in opposite direction when vestibular feedback was reversed compared with normal vestibular feedback for each stimulus polarity. Similar responses were observed in the group data (Fig. 6B). The mean peak sway was similar across the normal and reversed vestibular feedback conditions for each stimulus polarity (ARCL: $0.62 \pm$ 0.36 vs $0.80 \pm 0.40^{\circ}, t_{(7)}=1.23, p=0.260$; ALCR: $-0.66 \pm 0.51$ vs $-0.83 \pm 0.68^{\circ}, t_{(7)}=1.08, p=0.380$; Fig. $\left.6 C\right)$. In contrast, the mean peak of the torque profile over the initial $\sim 0-1 \mathrm{~s}$, which acts to accelerate the body in the direction of the anode, was in the opposite direction across normal and reversed vestibular feedback conditions (ARCL: $3.3 \pm 3.9$ vs $-3.6 \pm 5.4 \mathrm{Nm}$, ALCR: $-2.89 \pm 2.61$ vs $4.9 \pm$ $5.2 \mathrm{Nm}$, both $Z=2.52, p=0.012$; Fig. $6 C$ ).

The SVS stimulus evoked significant muscle responses during trials with normal and reversed vestibular feedback for all subjects. Data from a single subject show that the bandwidth of coherence between the input SVS and rectified EMG spanned a similar range of frequencies $(\sim 0-20 \mathrm{~Hz})$ for both vestibular feedback conditions (Fig. 7A). Comparison of the evoked muscle responses across balancing conditions, however, revealed inverted responses: during normal vestibular feedback, the muscle response exhibited an initial facilitation followed by an inhibition, whereas during the reversed vestibular feedback, an initial inhibition followed by facilitation was observed (Fig. 7A, arrows). Furthermore, with reversed vestibular feedback, the evoked muscle response was delayed; the first and second peaks occurred 30 and $70 \mathrm{~ms}$ later, respectively, relative to the equivalent peaks in the condition with normal vestibular feedback. A small facilitatory peak was observed early $(55 \mathrm{~ms})$ in the evoked muscle response when vestibular feedback was reversed, but remained below the $95 \%$ confidence interval. Finally, with reversed vestibular feedback, the magnitude of both coherence and the 

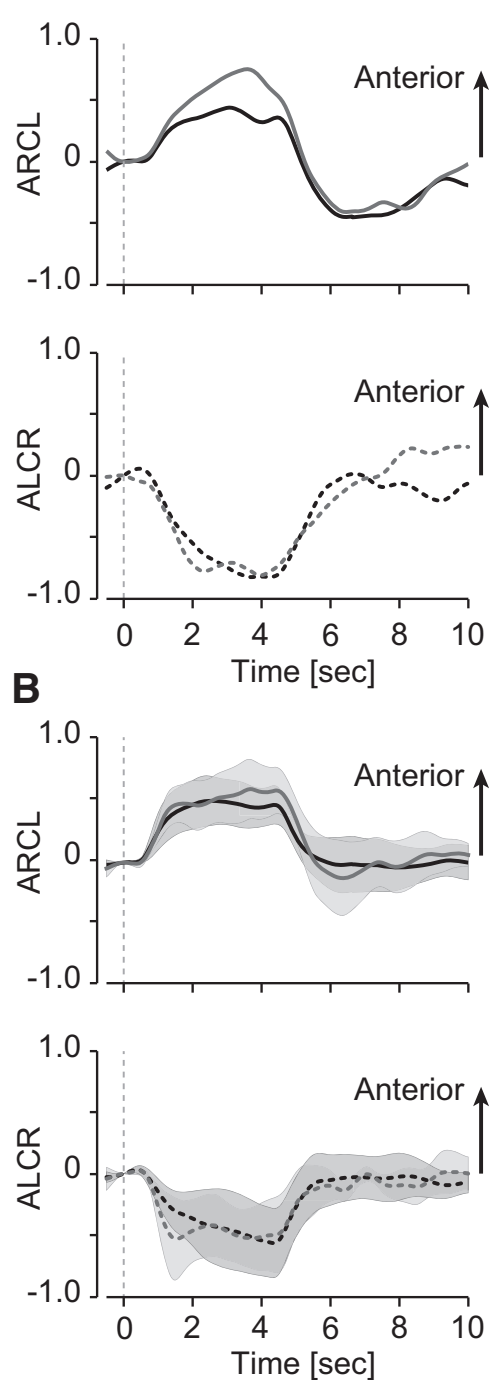

C

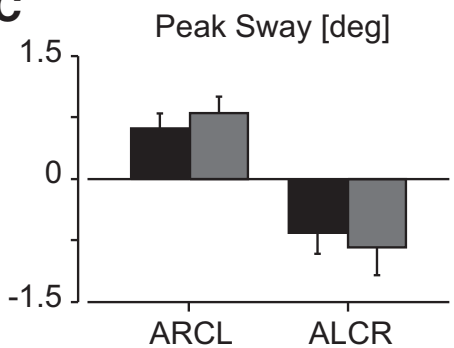

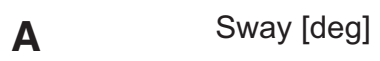

Moment [Nm]
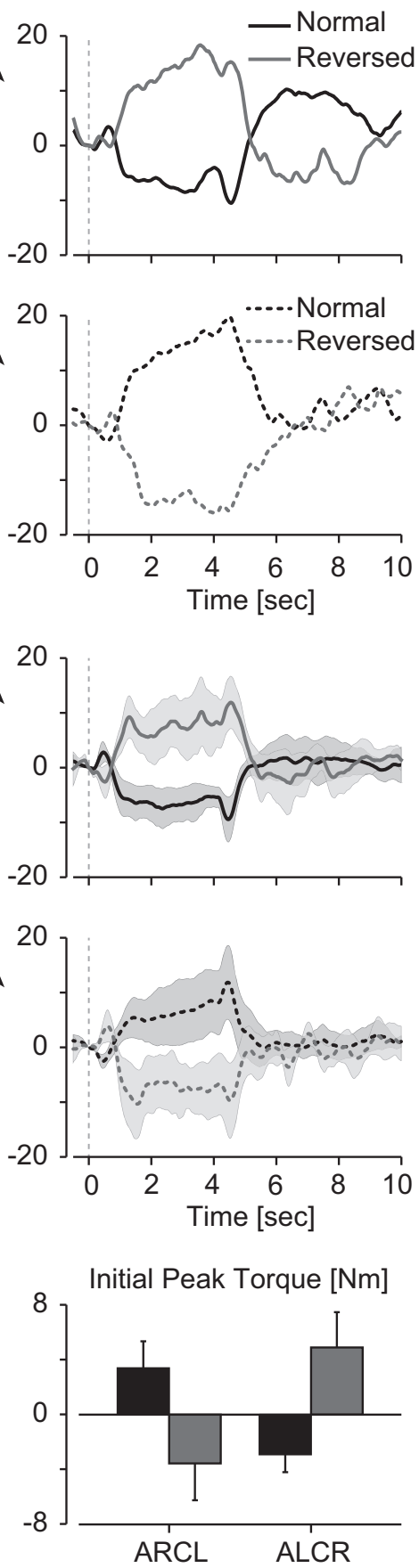

Figure 6. GVS-evoked body sway (left) and ankle moment (right) responses during balance conditions with normal and reversed vestibular feedback. Data are shown for a single subject $(\boldsymbol{A})$ and group means $(n=8)(\boldsymbol{B})$. Responses to each stimulus polarity (i.e., $A R C L$ and $A L C R$ ) are plotted separately. Body sway is in the same direction across normal and reversed conditions for each stimulus, whereas ankle torque is reversed. Shaded regions around the group mean represent the $95 \%$ confidence interval for the sway and ankle moment. Vertical lines indicate the onset of the $4 \mathrm{~s}$ square-wave electrical stimulus. Data from group mean $(n=8)$ peak sway and peak initial torque are also shown (C) for normal (black) and reversed vestibular feedback (gray) during both stimulus polarities. Error bars indicate the $95 \%$ confidence interval. evoked muscle response were reduced, which for the latter decreased by $40 \%$ relative to normal balance conditions.

The reversal, delay, and reduction in SVS-evoked muscle responses were reflected in the data for all subjects (Fig. $7 B$ ). With normal vestibular feedback, SVS evoked positive, shortlatency peaks at $62 \pm 4 \mathrm{~ms}$ and negative, medium-latency peaks at $105 \pm 8 \mathrm{~ms}$, whereas with reversed vestibular feedback, SVS evoked negative, short-latency peaks at $101 \pm 20 \mathrm{~ms}$ and positive, medium-latency peaks at $171 \pm 26 \mathrm{~ms}$ (Fig. $7 B$, arrows). An early facilitatory peak with reversed vestibular feedback was also observed in the mean evoked muscle response; however, this peak was small and did not exceed the 95\% confidence interval for six of eight subjects. Finally, coherence decreased with reversed vestibular feedback for all subjects and the amplitude of the evoked muscle response decreased on average by $47 \pm 24 \%\left(t_{(7)}=\right.$ $3.65, p=0.008)$. This result may be partially explained by the increased sway, which can swamp the evoked response due to swayrelated vestibular signals or volitional contributions (Fitzpatrick et al., 1994; Bacsi and Colebatch, 2005; Héroux et al., 2015), and the additional cutaneous input (Muise et al., 2012) caused by the toe-up orientation of the foot $\left(\sim 3.6^{\circ}\right.$ from horizontal $)$ that was adopted to maintain normal ankle somatosensory information when vestibular feedback was reversed.

We confirmed the observed timing changes (inversion and delay) in the evoked muscle response across normal and reversed vestibular feedback by plotting the mean phase-frequency responses (Fig. 8). With normal vestibular feedback phase decreased progressively from 0 rads starting at $1 \mathrm{~Hz}$. A similar progressive decrease in phase was observed with reversed vestibular feedback. In addition, phase responses were shifted by $\sim-180^{\circ}$ ( $\pi$ rads) at $1 \mathrm{~Hz}$ and the phase decreased with a steeper slope relative to normal vestibular feedback, suggesting an increased delay in the evoked muscle response. To examine the effect of a delay on the phase-frequency response, we also plotted responses with normal vestibular feedback, modified to be inverted and delayed by the difference in timing between the mean short-latency peaks obtained from conditions with normal and reversed vestibular feedback (39 ms). Responses from this inverted and delayed normal vestibular feedback condition were similar to responses obtained during the reversed vestibular feedback condition (Fig. 8), further suggesting that, with reversed vestibular feedback, the vestibular-evoked muscle response was inverted and delayed by 〜30-60 ms (Fig. 7).

\section{Discussion}

The aim of this study was to determine whether the transformation of vestibular signals for controlling human standing accounts for the direction of the vestibular error with respect to the contribution of muscle actions correcting the vestibular-evoked postural distur- 
bance. When subjects balanced in a single plane of motion, vestibular-evoked muscle responses were maximized when the direction of the vestibular disturbance was aligned with the direction of balance stabilization and decreased to zero as the directions became orthogonal. In addition, when the vestibular consequences of the ankle torques required to stand were reversed, the direction of vestibular-evoked ankle compensatory responses were inverted. In this reversed balance condition, however, the requisite vestibular transformation came at the cost of additional processing time. Ultimately, both results indicate that the nervous system organizes balance responses to counteract vestibular signals of head motion according to a muscle's ability to stabilize the body along the direction of vestibularimposed motion.

\section{Vestibular-evoked muscle responses indicate a muscle's contribution to balance along the vestibular error direction}

When subjects stood in a robotic balance system limited to move in the anteroposterior plane, vestibular-evoked muscle responses were largest when the vestibular error vector was parallel with the balance direction (i.e., head-left) and near zero when it was orthogonal to the balance direction (i.e., head-forward). This result is similar to the task dependency of vestibular-evoked responses in appendicular muscles: the influence of vestibular stimulation is only observed when vestibular information is relevant to the process of balancing the body (Britton et al., 1993; Fitzpatrick et al., 1994; Luu et al., 2012; Forbes et al., 2015). Based on this requirement, one could predict that responses would be evoked when the direction of balance was orthogonal to the error vector because the muscles were engaged in balancing the body and the vestibular afferents were activated by the electrical stimulus. Our results clarify this task dependence and reveal that the response is dependent, not only on the muscle's engagement in balance, but more specifically, upon the relevance of the muscle's contribution to correct balance for the vestibular error. Accordingly, with the head forward, a response was only observed when subjects balanced in the mediolateral plane because the vestibular error was aligned with the direction of balance. This further indicates that, although the soleus has the capacity to respond to mediolateral vestibular errors with the head forward, it does not when subjects balanced only in the anteroposterior plane because any correction to the orthogonal vestibular error signal is irrelevant to the balance task.

The presence of vestibular-evoked responses during mediolateral trials with the head forward also indicates that soleus muscles contribute to corrective responses evoked by the mediolaterally directed signals of head motion. This confirms findings that soleus muscles respond to mediolateral vestibular input when freely standing (Nashner and Wolfson, 1974; Day et al., 1997; Dakin et al., 2007) and aligns with recent observations that soleus motor units modulate their firing with mediolateral torque production in standing (Héroux et al., 2014). The difficulty with interpreting responses during free standing, however,
Coherence [-]

Cumulant density [-]
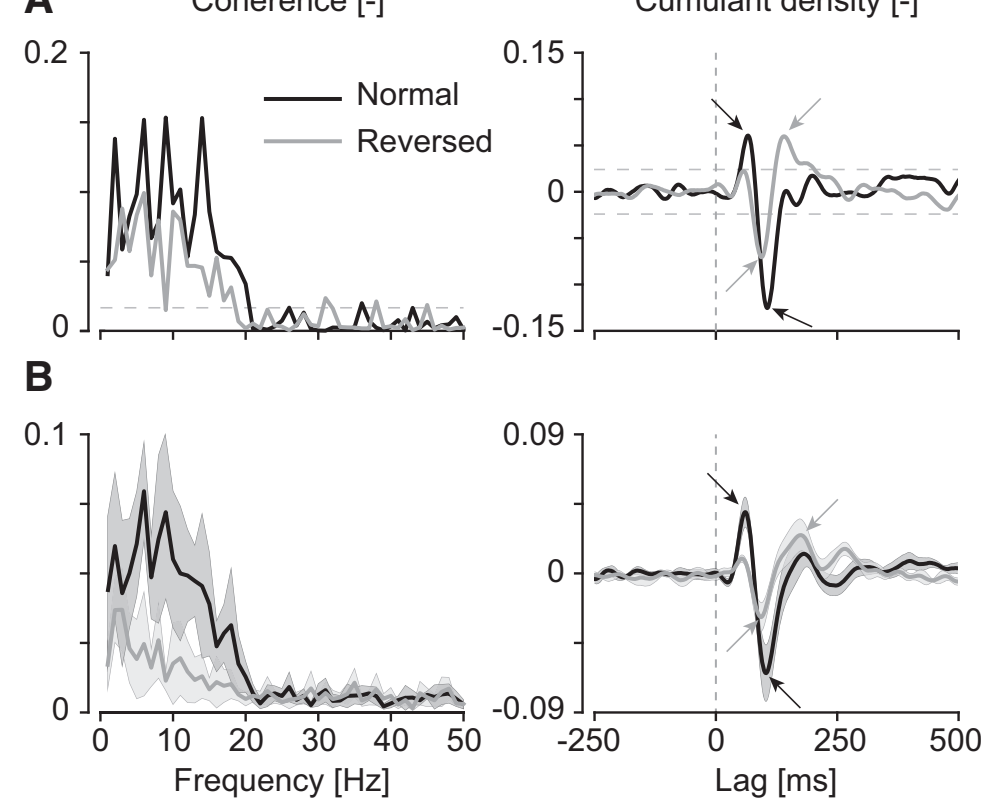

Frequency $[\mathrm{Hz}]$ Figure 7. Vestibular-evoked muscle responses with normal and reversed vestibular consequences of ankle motor actions. Data
are shown for a single subject $(\boldsymbol{A})$ and group means $(n=8)(\boldsymbol{B})$. The reversed condition has lower coherence and cumulant density

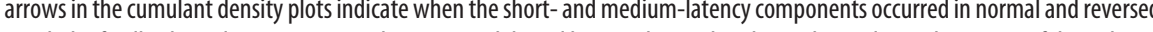

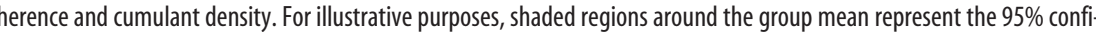
dence intervals for the coherence and cumulant density. Vertical lines in the cumulant density plots indicate the zero lag mark between the SVS and muscle activity.

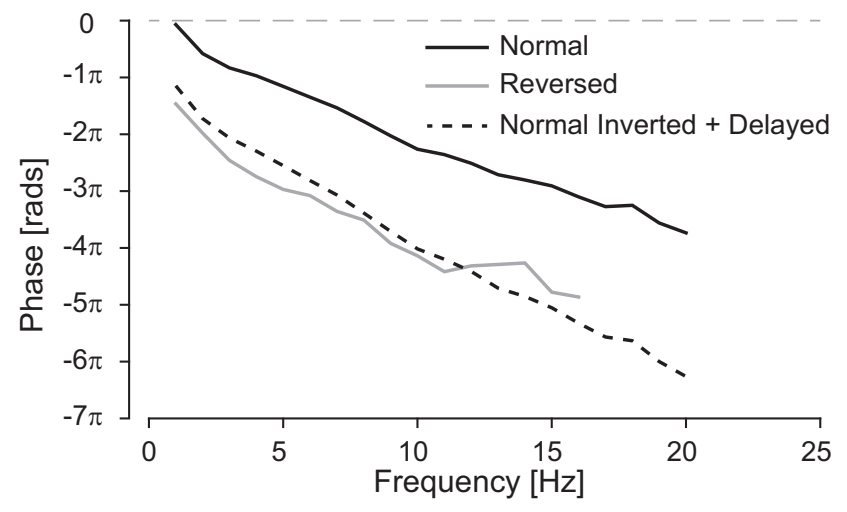

Figure 8. Group mean $(n=8)$ phase estimates between the SVS and muscle activity across the bandwidth of significant coherence. The "normal inverted + delayed" response (dotted line) was generated by using an inverted and delayed (39 ms) EMG signal when vestibular feedback was normal (solid black line). These phase estimates confirm that the vestibularevoked muscle responses during the reversed vestibular feedback balance condition are inverted and delayed relative to normal standing balance.

is that both feet generate ground reaction forces to balance the body, so a single muscle can contribute to body sway along axes not aligned with the biomechanical action of that muscle relative to its own joint. Our results suggest that the nervous system accounts for these mechanical changes and scales evoked responses according to the muscle's involvement in correcting for imposed vestibular errors.

\section{Reversed vestibular consequences of ankle motor torque}

Reversing the vestibular consequences of balance motor actions inverted motor responses evoked by electrical vestibular stimu- 
lation. During square-wave (GVS) trials, the initial ( $0-1$ s) torque peak was similar in magnitude but inverted across our two conditions (normal vs reversed) for both stimulus polarities. This inverted ankle torque (i.e., the net output of muscle responses that are also inverted) during the reversed condition ensured that, similar to normal balance conditions, corrective sway was in the direction of the anode. Similar reversals in vestibulomotor control have been observed in human (and animal) vestibuloocular reflexes (VORs) when exposed to optical reversals of vision using head-mounted prisms (Gonshor and Jones, 1976; Jones, 1977; Berthoz et al., 1981). VOR phase lags increase over the first week of exposure and reach $130^{\circ}$ at the end of the second, approximating a functional reversal of the reflex. The duration of this VOR adaptation, however, contrasts with the vestibulomotor reversals observed here, which developed rapidly. The majority of subjects were capable of balancing the robot within $30 \mathrm{~s}$, aligning with short adaptation timescales $(\sim 4 \mathrm{~min})$ reported previously for the human vestibular control of balance (Héroux et al., 2015). This implies that the nervous system can swiftly reassociate the new vestibular consequences of motion with motor signals generated to maintain balance. This rapid sensorimotor reassociation is essential to staying upright when transitioning between situations with altered relationships of motor commands and sensory feedback (e.g., stepping from shore to a stand-up paddleboard).

Re-associations of sensory and motor signals may be explained by conceptualizing the balance system as acting through its own reafference principle and forward sensory models. The balance system is thought to possess an internal model of the standing body's dynamics to predict the sensory consequences of desired postural motor actions (van der Kooij et al., 2001; Luu et al., 2012). When subjects balance with reversed vestibular feedback, the internal model is updated by comparing the model's sensory prediction of motor actions with actual sensory feedback and proficient balance behavior is restored after a brief period of exposure ( $\sim 30-90$ s). It has been proposed recently that vestibular networks contribute to this rapid internal model update for standing balance (Héroux et al., 2015). Results from our reversed condition support this proposal because vestibular-evoked muscle (and torque) responses were inverted and phase-frequency plots shifted by at least $180^{\circ}$ at all frequencies. Our results establish that the internal model of balance can adapt its motor output to accommodate reversed relationships between vestibular afference and balance motor commands. Such mechanisms provide postural muscles with the flexibility to counteract a balance disturbance under conditions that change the sensory consequences of balancing dramatically compared with over ground upright standing.

\section{Neural integration and central processing of a transformed vestibulomotor relationship during balance}

This study provides evidence that vestibular-evoked muscle activity represents a flexible and organized balance response to counteract vestibular-induced postural disturbances. The soleus responded only to the component of the net vestibular error aligned with and relevant to the balance direction, not to the cumulative vestibular afferent activity evoked by the electrical stimulus, as would be expected of a vestibulospinal reflex loop. Further support that vestibular-evoked responses represent an organized balance response comes from single motor unit responses in soleus muscles to vestibular stimuli, which exhibit prolonged changes in motor unit discharge rather than the shortduration reflexive responses elicited by other forms of neural stimulation (Dakin et al., 2016). The vestibular system is well suited to mediate transformed vestibulomotor relationships because the vestibular nuclei receive input from regions within the cerebellum that implement coordinate transformations of vestibular and somatosensory information (Kleine et al., 2004; Shaikh et al., 2004) to estimate body movement in space (Mergner et al., 1991; Brooks and Cullen, 2009).

The flexibility of these organized balance responses can, however, come at the cost of additional processing time: the inverted polarity of vestibular-evoked responses in the reversed vestibular feedback trial was accompanied by an additional delay $(\sim 38-60$ $\mathrm{ms}$ ). This suggests the involvement of additional neural processing for the spatial transformation of vestibular signals. Longer than expected response latencies are inherent features of vestibular-evoked muscle responses and occur $30 \mathrm{~ms}$ later than those elicited by magnetic cortex stimulation (Britton et al., 1993), even though signals evoking each type of response presumably travel via neural pathways with similar conduction velocities. This delay is also observed in single motor unit responses (Dakin et al., 2016) and supports the likelihood that the delayed vestibular-evoked responses observed here are due to the neural integration and central processing involved in transforming the vestibular error signal (Britton et al., 1993). Additional neural processing time has also been reported after adaptation of VORs to magnifying glasses (Miles and Eighmy, 1980; Ramachandran and Lisberger, 2005), a process thought to involve plasticity of the neural circuits projecting on interneurons contributing to the VOR (Miles et al., 1980; Lisberger et al., 1994). We propose that additional delays in sensorimotor pathways represent one solution for the nervous system to transform vestibular signals effectively for movement control.

\section{Conclusions}

In conclusion, vestibular-evoked muscle responses are transformed with head orientation depending upon the relative alignment between the direction of a vestibular error and the relevance of a muscle's contribution to counteract the vestibular error. Furthermore, the polarity of evoked muscle and torque responses are inverted when the relationship between vestibular afference and the motor actions required to stand is reversed. These results indicate that corrective motor commands accommodate for the muscle's adapted contribution such that the associated sway remains compensatory to the direction of the postural disturbance. Our study provides insight into the central integration and processing associated with vestibular transformations that are essential to standing upright.

\section{References}

Bacsi AM, Colebatch JG (2005) Evidence for reflex and perceptual vestibular contributions to postural control. Exp Brain Res 160:22-28. CrossRef Medline

Berthoz A, Jones GM, Bégué AE (1981) Differential visual-adaptation of vertical canal-dependent vestibulo-ocular reflexes. Exp Brain Res 44:1926. Medline

Britton TC, Day BL, Brown P, Rothwell JC, Thompson PD, Marsden CD (1993) Postural electromyographic responses in the arm and leg following galvanic vestibular stimulation in man. Exp Brain Res 94:143-151. Medline

Brooks JX, Cullen KE (2009) Multimodal integration in rostral fastigial nucleus provides an estimate of body movement. J Neurosci 29:1049910511. CrossRef Medline

Cathers I, Day BL, Fitzpatrick RC (2005) Otolith and canal reflexes in human standing. J Physiol 563:229-234. CrossRef Medline

Cenciarini M, Peterka RJ (2006) Stimulus-dependent changes in the vestibular contribution to human postural control. J Neurophysiol 95:27332750. CrossRef Medline 
Dakin CJ, Son GM, Inglis JT, Blouin JS (2007) Frequency response of human vestibular reflexes characterized by stochastic stimuli. J Physiol 583: 1117-1127. CrossRef Medline

Dakin CJ, Luu BL, van den Doel K, Inglis JT, Blouin JS (2010) Frequencyspecific modulation of vestibular-evoked sway responses in humans. J Neurophysiol 103:1048-1056. CrossRef Medline

Dakin CJ, Inglis JT, Blouin JS (2011) Short and medium latency muscle responses evoked by electrical vestibular stimulation are a composite of all stimulus frequencies. Exp Brain Res 209:345-354. CrossRef Medline

Dakin CJ, Dalton BH, Luu BL, Blouin JS (2014) Rectification is required to extract oscillatory envelope modulation from surface electromyographic signals. J Neurophysiol 112:1685-1691. CrossRef Medline

Dakin CJ, Héroux ME, Luu BL, Inglis JT, Blouin JS (2016) Vestibular contribution to balance control in the medial gastrocnemius and soleus. J Neurophysiol 115:1289-1297. CrossRef Medline

Day BL, Cole J (2002) Vestibular-evoked postural responses in the absence of somatosensory information. Brain 125:2081-2088. CrossRef Medline

Day BL, Fitzpatrick RC (2005) Virtual head rotation reveals a process of route reconstruction from human vestibular signals. J Physiol 567:591597. CrossRef Medline

Day BL, Séverac Cauquil A, Bartolomei L, Pastor MA, Lyon IN (1997) Human body-segment tilts induced by galvanic stimulation: A vestibularly driven balance protection mechanism. J Physiol 500:661-672. CrossRef Medline

Fitzpatrick RC, Day BL (2004) Probing the human vestibular system with galvanic stimulation. J Appl Physiol 96:2301-2316. Medline

Fitzpatrick R, Burke D, Gandevia SC (1994) Task-dependent reflex responses and movement illusions evoked by galvanic vestibular stimulation in standing humans. J Physiol 478:363-372. CrossRef Medline

Forbes PA, Dakin CJ, Geers AM, Vlaar MP, Happee R, Siegmund GP, Schouten AC, Blouin JS (2014) Electrical vestibular stimuli to enhance vestibulo-motor output and improve subject comfort. PLoS One 9:e84385. CrossRef Medline

Forbes PA, Siegmund GP, Schouten AC, Blouin JS (2015) Task, muscle and frequency dependent vestibular control of posture. Front Integr Neurosci 8:94. CrossRef Medline

Goldberg JM, Smith CE, Fernández C (1984) Relation between discharge regularity and responses to externally applied galvanic currents in vestibular nerve afferents of the squirrel-monkey. J Neurophysiol 51:12361256. Medline

Gonshor A, Jones GM (1976) Extreme vestibulo-ocular adaptation induced by prolonged optical reversal of vision. J Physiol 256:381-414. CrossRef Medline

Halliday DM, Rosenberg JR, Amjad AM, Breeze P, Conway BA, Farmer SF (1995) A framework for the analysis of mixed time series/point process data: theory and application to the study of physiological tremor, single motor unit discharges and electromyograms. Prog Biophys Mol Biol 64: 237-278. CrossRef Medline

Héroux ME, Dakin CJ, Luu BL, Inglis JT, Blouin JS (2014) Absence of lateral gastrocnemius activity and differential motor unit behavior in soleus and medial gastrocnemius during standing balance. J Appl Physiol 116:140148. CrossRef Medline

Héroux ME, Law TC, Fitzpatrick RC, Blouin JS (2015) Cross-modal calibration of vestibular afference for human balance. PLoS One 10:e0124532. CrossRef Medline

Iles JF, Pisini JV (1992) Vestibular-evoked postural reactions in man and modulation of transmission in spinal reflex pathways. J Physiol 455:407424. CrossRef Medline

Jones GM (1977) Plasticity in the adult vestibulo-ocular reflex arc. Philos Trans R Soc Lond B Biol Sci 278:319-334. CrossRef Medline

Kim J, Curthoys IS (2004) Responses of primary vestibular neurons to galvanic vestibular stimulation (GVS) in the anaesthetised guinea pig. Brain Res Bull 64:265-271. CrossRef Medline

Kleine JF, Guan Y, Kipiani E, Glonti L, Hoshi M, Büttner U (2004) Trunk position influences vestibular responses of fastigial nucleus neurons in the alert monkey. J Neurophysiol 91:2090-2100. CrossRef Medline

Lisberger SG, Pavelko TA, Broussard DM (1994) Neural basis for motor learning in the vestibuloocular reflex of primates. I. Changes in the responses of brain-stem neurons. J Neurophysiol 72:928-953.
Loram ID, Lakie M (2002) Direct measurement of human ankle stiffness during quiet standing: the intrinsic mechanical stiffness is insufficient for stability. J Physiol 545:1041-1053. CrossRef Medline

Lund S, Broberg C (1983) Effects of different head positions on postural sway in man induced by a reproducible vestibular error signal. Acta Physiol Scand 117:307-309. CrossRef Medline

Luu BL, Huryn TP, Van der Loos HF, Croft EA, Blouin JS (2011) Validation of a robotic balance system for investigations in the control of human standing balance. IEEE Trans Neural Syst Rehabil Eng 19:382-390. CrossRef Medline

Luu BL, Inglis JT, Huryn TP, Van der Loos HF, Croft EA, Blouin JS (2012) Human standing is modified by an unconscious integration of congruent sensory and motor signals. J Physiol 590:5783-5794. CrossRef Medline

Marsden JF, Castellote J, Day BL (2002) Bipedal distribution of human vestibular-evoked postural responses during asymmetrical standing. J Physiol 542:323-331. CrossRef Medline

Mergner T, Siebold C, Schweigart G, Becker W (1991) Human perception of horizontal trunk and head rotation in space during vestibular and neck stimulation. Exp Brain Res 85:389-404. Medline

Mian OS, Day BL (2014) Violation of the craniocentricity principle for vestibularly evoked balance responses under conditions of anisotropic stability. J Neurosci 34:7696-7703. CrossRef Medline

Mian OS, Dakin CJ, Blouin JS, Fitzpatrick RC, Day BL (2010) Lack of otolith involvement in balance responses evoked by mastoid electrical stimulation. J Physiol 588:4441-4451. CrossRef Medline

Miles FA, Eighmy BB (1980) Long-term adaptive-changes in primate vestibuloocular reflex. 1. Behavioral observations. J Neurophysiol 43:14061425. Medline

Miles FA, Fuller JH, Braitman DJ, Dow BM (1980) Long-term adaptivechanges in primate vestibuloocular reflex. 3. Electro-physiological observations in flocculus of normal monkeys. J Neurophysiol 43:1437-1476.

Muise SB, Lam CK, Bent LR (2012) Reduced input from foot sole skin through cooling differentially modulates the short latency and medium latency vestibular reflex responses to galvanic vestibular stimulation. Exp Brain Res 218:63-71. CrossRef Medline

Nashner LM, Wolfson P (1974) Influence of head position and proprioceptive cues on short latency postural reflexes evoked by galvanic stimulation of human labyrinth. Brain Res 67:255-268. CrossRef Medline

Pastor MA, Day BL, Marsden CD (1993) Vestibular induced postural responses in Parkinson's disease. Brain 116:1177-1190. CrossRef Medline

Peters RM, Rasman BG, Inglis JT, Blouin JS (2015) Gain and phase of perceived virtual rotation evoked by electrical vestibular stimuli. J Neurophysiol 114:264-273. CrossRef Medline

Pintelon R, Schoukens J (2012) System identification: a frequency domain approach, Ed 2. New York: IEEE.

Pospisil ER, Luu BL, Blouin JS, Van der Loos HF, Croft EA (2012) Independent ankle motion control improves robotic balance simulator. Conf Proc IEEE Eng Med Biol Soc 2012:6487-6491. CrossRef Medline

Ramachandran R, Lisberger SG (2005) Normal performance and expression of learning in the vestibulo-ocular reflex (VOR) at high frequencies. J Neurophysiol 93:2028-2038. Medline

Reynolds RF (2010) The effect of voluntary sway control on the early and late components of the vestibular-evoked postural response. Exp Brain Res 201:133-139. CrossRef Medline

Reynolds RF (2011) Vertical torque responses to vestibular stimulation in standing humans. J Physiol 589:3943-3953. CrossRef Medline

Shaikh AG, Meng H, Angelaki DE (2004) Multiple reference frames for motion in the primate cerebellum. J Neurosci 24:4491-4497. CrossRef Medline

St George RJ, Day BL, Fitzpatrick RC (2011) Adaptation of vestibular signals for self-motion perception. J Physiol 589:843-853. CrossRef Medline

Tribukait A, Rosenhall U (2001) Directional sensitivity of the human macula utriculi based on morphological characteristics. Audiol Neurootol 6:98-107. CrossRef Medline

van der Kooij H, Jacobs R, Koopman B, van der Helm F (2001) An adaptive model of sensory integration in a dynamic environment applied to human stance control. Biol Cybern 84:103-115. CrossRef Medline

Wardman DL, Taylor JL, Fitzpatrick RC (2003) Effects of galvanic vestibular stimulation on human posture and perception while standing. J Physiol 551:1033-1042. CrossRef Medline 